\title{
Ordre public et poursuites criminelles sous le
} Directoire (1795-1799)

L'expérience d'un modèle judiciaire libéral

Public Order and Criminal Prosecution under the Directory (1795-1799). The

Experience of a Liberal Judicial Model

\section{Emmanuel Berger}

\section{OpenEdition}

Journals

Édition électronique

URL : https://journals.openedition.org/ahrf/11274

DOI : 10.4000/ahrf.11274

ISSN : 1952-403X

Éditeur :

Armand Colin, Société des études robespierristes

Édition imprimée

Date de publication : 1 décembre 2007

Pagination : 135-152

ISSN : 0003-4436

\section{Référence électronique}

Emmanuel Berger, «Ordre public et poursuites criminelles sous le Directoire (1795-1799)», Annales historiques de la Révolution française [En ligne], 350 | octobre-décembre 2007, mis en ligne le 01 janvier 2011, consulté le 01 juillet 2021. URL : http://journals.openedition.org/ahrf/11274 ; DOI : https:// doi.org/10.4000/ahrf.11274 


\title{
ORDRE PUBLIC ET POURSUITES CRIMINELLES SOUS LE DIRECTOIRE (1795-1799). L'EXPÉRIENCE D'UN MODĖLE JUDICIAIRE LIBÉRAL*
}

\author{
Emmanuel BERGER
}

\begin{abstract}
Au cours des premières années de la Révolution, les Constituants instaurent un nouveau modèle judiciaire. Ce dernier, que l'on peut qualifier de libéral, est destiné à réaliser la synthèse entre la nécessité de protéger les libertés et le devoir de maintenir l'ordre public. Cependant, dès 1790, certains députés contestent la capacité de la justice libérale à protéger la population face aux attaques des brigands et des criminels. Le laxisme des juges de paix et les "acquittements scandaleux " prononcés par les jurés populaires en sont tenus pour responsables. Les attaques persistent tout au long du Directoire et deviennent dominantes à partir du Consulat, au point de provoquer la suppression progressive de plusieurs garanties essentielles aux droits naturels des citoyens. À travers la problématique des poursuites criminelles menées sous le Directoire, nous avons tenté de comprendre le fondement et les implications des réformes consulaires et impériales qui provoquèrent la disparition du modèle judiciaire de la Révolution.
\end{abstract}

Mots-clés : justice pénale, ordre public, poursuites criminelles, juge de paix, jury d'accusation, libertés individuelles.

Au cours des années 1790-1791, l'Assemblée constituante opère une révolution judiciaire profonde. En l'espace de quelques mois, les députés remplacent la justice d'Ancien Régime par un nouveau modèle judiciaire.

*Cette publication a été rédigée dans le cadre du Pôle d'attraction interuniversitaire P6/01, " Justice et société : histoire sociopolitique de la justice en Belgique (1795-2005) ", Politique scientifique fédérale belge. 
Ce modèle est perçu comme libéral dans la mesure où il est fondé sur la protection des libertés individuelles des citoyens. Celles-ci sont garanties par plusieurs principes tels que l'indépendance de la justice, l'élection des juges, l'institution de jurys au niveau de la mise en accusation et du jugement des crimes, la division du ministère public entre juges élus et nommés, la délégation du pouvoir de poursuite aux seuls juges élus, la procédure pénale accusatoire ou encore la légalité et la fixité des peines.

Toutefois le nouveau modèle judiciaire fait l'objet de critiques dès 1790. Plusicurs Constituants parlent d'" expérience dangereuse "', d'《abstraction absolue $»^{2}$, de " dangereuse rêverie $»^{3}$ ou de «système métaphysique » pensé en philosophe et non en magistrat". On conteste en réalité la capacité de la justice libérale à maintenir l'ordre public et plus précisément à protéger la population face aux attaques des brigands et des criminels. Les reproches persistent tout au long du Directoire et deviennent dominants sous le Consulat.

À partir de l'an VIII, le gouvernement entreprend, avec l'appui d'une partie des législateurs, une déconstruction progressive du modèle judiciaire de 1791. La nouvelle Constitution consulaire réunifie notamment le ministère public au profit du pouvoir exécutif. Cette politique réformatrice trouve son aboutissement à la suite de l'attentat de la rue SaintNicaise perpétré le 4 nivôse an IX contre Bonaparte. Dans un climat d'insécurité, deux lois importantes sont votées en l'espace de quelques jours. La loi du 7 pluviôse an IX confie la poursuite des infractions au gouvernement tandis que la loi du 18 pluviôse crée une juridiction d'exception destinée à juger les crimes les plus graves et à contourner la justice ordinaire : le tribunal criminel spécial'. Au cours des débats, le modèle judiciaire de la Révolution est fortement décrié et considéré comme une « théorie $»^{6}$ composée de « formes abstraites ${ }^{7}$, de « combinaisons métaphysiques $»^{8}$ et de " maximes rigides ${ }^{4}$, dont les défenseurs doivent être qualifiés de

(1) Archives parlementaires (ci-après $A P$ ), $1^{\text {tore }}$ série, t. XVII, 10 août 1790 , discours de l'Abbé Maury, p. 699.

(2) $A P, 1^{\text {iee }}$ série, $\mathrm{t}$ XXI, 26 décembre 1790, discours de Baco de la Chapelle, p. 672.

(3) Idem.

(4) $A P, 1^{\text {trx }}$ séric, t. XXI, 27 décembre 1790, discours de Mougins, p. 682.

(5) La loi du 18 pluvióse an IX autorise le gouvernement à établir dans les départements où il les juge nécessaires des tribunaux criminels spéciaux jugeant sans jury. Ceux-ci sont composés de magistrats, de militaires et de civils nommés par le premier consul. Leur champ de compétence est large : vagabondage, évasion des condamnés, vol sur les grandes routes, rassemblement séditieux, assassinat prémédité, etc. En matière procédurale, les droits de la défense sont considérablement affaiblis : la mise en accusation est décidée par le tribunal spécial, le prévenu ne prend connaissance de la nature des charges que lors du procès, les jugements sont sans appel, etc. (Robert ALLFN, Les tribunatax criminels sous la Révolution et l'Empire. 1792-1811, Rennes, PUR, 2005, p. 256-266).

(6) $A P, 2^{c}$ série, t. II, 1" pluviôse an IX, discours de Challan, p. 122.

(7) $A P, 2^{\star}$ série, t. II, 29 nivôse an IX, discours de Duveyrier, p. 107.

(8) Idem.

(9) $A P, 2^{c}$ série, t. II, 11 pluviôse an IX, discours de Laussat, p. 257. 
"philosophe $"^{10}$ ou de "moraliste $"$ ". Lors de la séance du 18 pluviôse an IX, l'orateur du gouvernement, Français de Nantes, porte l'attaque la plus virulente :

«Que l'on descende, pour un moment, des sommités nébuleuses de ces théories (où l'on est tellement élevé qu'on n'aperçoit plus rien de ce qui se passe sur la terre) dans les prisons où sont entassés ces êtres féroces [...] Ils n'ont pas seulement cessé d'appartenir à la cité, mais encore à l'espèce humaine. Ce n'est pas avec de la métaphysique ni d'ingénieuses hypothèses que vous purgerez les grandes routes, que vous inspirerez la confiance des voyageurs, que vous rassurerez le repos des familles. N'est-ce donc pas assez la rude expérience que nous avons faite pendant dix ans sur l'abus de ces abstractions, qui supposent tous les hommes bons, sensibles, désintéressés, sans vouloir commencer un nouveau cours de ce genre ? Et n'est-il pas temps de revenir aux maximes pratiques d'un sage gouvernement, qui veut s'assurer la paix de tous les citoyens par la punition de tous les assassins ? $»^{12}$

Ces critiques occultent cependant l'importance que les Constituants accordent au maintien de l'ordre public lorsqu'ils créent le nouveau modèle judiciaire. Exception faite de quelques envolées lyriques de certains orateurs, ses partisans font preuve de pragmatisme. Le système proposé se base sur une réflexion critique des expériences étrangères et de l'Ancien Régime. On n'hésite pas en outre à prendre des mesures transitoires qui, bien qu'en contradiction avec les principes du modèle judiciaire de la Révolution, sont nécessaires à son établissement et à sa viabilité ${ }^{13}$.

En réalité, les Constituants considèrent le maintien de l'ordre public et la sécurité des citoyens comme un objectif essentiel de la police et de la justice. Lorsque le député Adrien Duport présente le rapport sur la nouvelle procédure criminelle, le 27 novembre 1790, il débute son discours en proclamant le maintien de l'ordre public comme le « premier besoin des peuples policés ${ }^{14}$. Thouret fait part du même intérêt et rappelle dans ses interventions que la principale préoccupation de la nation est le « rétablissement de l'ordre public », sans lequel " la Constitution ne peut pas prospérer " 19 . Toutefois, selon le même Thouret, l'établissement de la tranquillité générale et l'exécution sûre et énergique des lois pénales ne doivent pas se faire au détriment des libertés individuelles. En d'autres termes, il ne faut pas que sous prétexte de protéger les sûretés individuelle et publique, on les détruise toutes les deux.

(10) $A P, 2$ série, t. Il, 7 pluviôse an IX, discours de Delpierre jeune (des Vosges), p. 220

(11) /dem.

(12) $A P, 2^{`}$ séric, t. II, 18 pluviôse an IX, discours de Français de Nantes, p. 327.

(13) C'est notamment le cas des gendarmes - des militaires - qui sont nommés officiers de police judiciaire aux cótés des juges de paix (art. 3 titre I section I de la loi des 16-29 septembre 1791).

(14) $A P^{\prime}, 1^{c c}$ séric, t. XXI, 27 novembre 1790, discours de Duport, p. 44.

(15) Ibid., t. XXI, 30 décembre 1790, discours de Thouret, p. 715. 
Il s'agit de la différence la plus marquée entre les modèles judiciaires révolutionnaire et napoléonien. Les législateurs de 1791 ne pensent pas que la protection des libertés individuelles soit antinomique au maintien de l'ordre public. Cette conception résulte de la peur exprimée à l'égard de la tyrannie et des abus commis par les pouvoirs judiciaire et exécutif sous l'Ancien Régime. Le modèle judiciaire libéral est par conséquent conçu de manière à neutraliser l'influence des deux pouvoirs et à empêcher qu'ils ne portent atteinte à la liberté individuelle des citoyens. La séparation du couple liberté-ordre public n'intervient qu'à la suite de l'empiètement progressif du pouvoir exécutif sur le pouvoir judiciaire. Afin de justifier cette ingérence anticonstitutionnelle, le Directoire n'aura de cesse d'invoquer la protection de l'ordre public et de regretter les entorses faites au modèle judiciaire libéral, tout en les considérant comme nécessaires. À partir de l'an VIII et de l'an IX, la volonté de puissance du gouvernement provoque peu à peu la soumission des autres pouvoirs et la limitation des libertés. Conscient de la rupture introduite par rapport aux principes de 1791 , le discours consulaire puis impérial vise, à travers le discrédit du modèle judiciaire révolutionnaire, à légitimer la subordination des libertés des citoyens au maintien de l'ordre public.

Dans ce mouvement de déconstruction de la justice de la Révolution, le Directoire occupe une place particulière du fait de l'attitude ambiguë que le régime entretient avec les institutions judiciaires. Alors que les législateurs de 1791 et de 1795 s'étaient accordés pour préserver l'indépendance de la justice par rapport au pouvoir exécutif, le Directoire cherche, tout au long de son existence, à contrôler l'appareil judiciaire. Comme la Constitution de l'an III ne le lui permet pas, il use d'autres moyens. Il essaie notamment de remplacer les mécanismes électifs par des mécanismes de nomination et il recourt aux lois d'exception pour modeler les élections en sa faveur. Au lendemain du coup d'État du 18 fructidor an V, les Conseils autorisent le Directoire non seulement à annuler les élections des départements mais également à pourvoir aux places vacantes dans l'ensemble du corps judiciaire, et ce, jusqu'aux élections de l'an VI. Dans les faits, le Directoire réussit à proroger la loi jusqu'aux élections de l'an VII ${ }^{16}$.

Cette volonté de contrôler l'issue des élections se double d'une volonté de mettre un terme à l'imprévisibilité des décisions des tribunaux ordinaires. Aussi, le Directoire confie-t-il aux conseils de guerre et aux commissions militaires le jugement de civils par l'intermédiaire de lois d'exception : la loi du 19 fructidor de l'an V sur les émigrés, la loi du 29 nivôse an VI sur le brigandage, la loi du 24 messidor an VII plus connue sous le nom de «loi des otages» et, son complément, la loi du 14 fructidor an VII. Droz, 1998.

(16) Jacques Logie, Les magistrats des cours et des tribunaux en Belgique (1794-1914), Genève, 
Par rapport à la législation de 1791, la période du Directoire se caractérise également par un durcissement du droit, nécessité par la recherche de moyens efficaces afin de diminuer une délinquance jugée dangereuse pour la société et l'État ${ }^{17}$. On assiste ainsi à la création d'un Ministère de la Police générale pour micux lutter contre les mendiants et les vagabonds mais aussi contre les adversaires politiques du gouvernement. La loi du 22 prairial an IV punit désormais toute tentative de crime comme le crime luimême. La loi du 26 floréal an V rétablit la peine de mort en matière de vol, mesure qui avait fait scandale à la fïn de l'Ancien Régime ${ }^{\text {ts. }}$.

Linflation de lois répressives et le recours aux juridictions d'exception révèlent l'échec des tentatives faites par le pouvoir exécutif pour réformer en profondcur l'ordre judiciaire. En effet, les législateurs veillent à voter des lois d'exception qui sont temporaires et doivent être obligatoirement renouvelées. Les principes du modèle judiciaire de la Révolution sont dès lors préservés et à nouveau appliqués lorsque l'ordre public est rétabli. Cette impression d'échec est accentuée par les multiples refus du Corps législatif de voter des réformes pénales réclamées par le gouvernement : le 9 pluviôse an V, les Anciens refusent de réduire les pouvoirs du jury d'accusation, au mois de ventôse an VI, le projet de résolution relatif à la surveillance et à la forfaiture des juges est écarté de l'ordre du jour par les Cinq-Cents, le 26 vendémiaire an VIII, les Anciens rejettent la résolution visant à pénaliser le vagabondage, etc.

Lincapacité du gouvernement à réformer durablement le modèle judiciaire libéral a été dénoncée par les détracteurs de ce dernier, comme la cause première de la prolifération du brigandage. Ces accusations tendent à rendre un certain nombre de principes et d'institutions judiciaires établies par les Constituants responsables de l'impunité du crime et de l'insécurité générale qui gagne la France. Toutefois, la permanence et l'analogie des reproches faits entre 1791 et le Consulat autorisent à douter de leur pertinence. La continuité des critiques révèle leur nature essentiellement politique fondée sur une conception autoritaire du mode de gouvernement et une vision sécuritaire de la justice.

Un tel parti-pris ne peut susciter que des appréciations négatives sur le modèle judiciaire de la Révolution et ne permet pas de conclure d'emblée à la faillite de ce dernier en matière de maintien de l'ordre public. Aussi, nous efforcerons-nous de réévaluer sereinement la capacité du modèle judiciaire établi en 1791 et prolongé en 1795 à assurer la sécurité de la population. Pour ce faire, nous analyserons le fonctionnement,

(17) Jean-Louis Halpérin, “ Continuité et rupture dans l'évolution de la procédurc pénale en France de 1795 à 1810 ", dans Xavjer RousSEauX, Marie-Sylvie DuPONT-Bouchait, Claude Vall, dir., Révolutions et justice pénale en Europe. Modeles français et traditions nationales (1730-1830), Paris, LHarmattan, 1999, p. J(19-130.

(18) Bernard SchNAPPER, "Les systèmes répressifs français de 1789 à 1815 ", Xavier RousSEAuX, Marie-Sylvie Dupont-Bouchat, Claude VAel, dir., op. cit., p. 17-36. 
à l'époque du Directoire, de deux phases essentielles de la procédure pénale qui firent l'objet des critiques les plus vives et furent en réaction réformées sous le Consulat et l'Empire : la poursuite dirigée par des officiers de police judiciaire élus et la mise en accusation prononcée par des jurés populaires.

La remise en cause du concours des citoyens à l'exercice de la justice constitue une rupture importante avec l'organisation judiciaire révolutionnaire. Lors de la fondation de cette dernière, sous la Constituante, la participation " populaire " représente en effet une innovation majeure destinée à protéger les droits naturels de chacun. Elle repose sur le postulat que le peuple étant la source de toute puissance, le pouvoir judiciaire ne doit pas lui échapper. Cette participation se traduit de manière indirecte par l'élection des juges et de manière immédiate par l'institution du jury.

Lélection temporaire des juges est conçue comme nécessaire afin d'empêcher que la fonction ne devienne une propriété qui rappellerait le corporatisme de l'Ancien Régime et qui rendrait les magistrats négligents, paresseux et jaloux de leurs prérogatives ${ }^{12}$. Lélection a également pour objet de soustraire les juges au pouvoir exécutif. Les Constituants craignent que leur nomination par le roi ou par ses ministres ne fasse renaitre le despotisme ou désigner les plus intrigants. Lors de la séance du 7 mai 1790, Roederer prononce un discours qui exprime cette crainte : soit l'institution royale des juges n'exercera aucune influence sur l'ordre judiciaire, mais alors pourquoi la réclamer? Soit elle aura une réelle incidence sur le cours de la justice, mais alors que deviendrait l'indépendance des magistrats face aux courtisans du prince? Le discours fait basculer l'Assemblée qui décrète que les juges seront élus pour une durée déterminée par le peuple et seront nommés par le roi sans que celui-ci puisse refuser le candidat unique issu de l'élection. En légitimant le suffrage populaire, les Constituants confortent " la régénération absolue " de l'organisation judiciaire" et adoptent une alternative exprimée, le 30 mars 1790, par Charles Chabroud :

« Sans doute, on ne doit pas imaginer que le peuple choisira toujours bien ; il ne faut pas compter sur des miracles. Mais je dis [...] qu'il n'y a pas de raison, à beaucoup près, de croire que les ministres du roi fissent mieux, je dis que l'amovibilité des juges sera le remède des erreurs du peuple ; et enfin je préfère que le blâme d'un mauvais choix ne puisse jamais remonter jusqu'au prince ${ }^{21}$.

(19) Guillaume Mf́tairıE, "L'électivité des magistrats judiciaires en France, entre Révolution et monarchies (1789-1814) ", dans Jacques KRYNEN, dir., L'élection des juges. Étude historique française et contemporaine, Paris, PUF, 1999, p. 21-65.

(20) $A P, 1^{\text {ert }}$ série, t. XII, 24 mars 1790 , discours de Thouret, p. 345.

(21) Ibid., t. XII, 30 mars 1790, discours de Chabroud, p. 446. 
La poursuite pénale aux mains des juges

La poursuite constitue une phase essentielle de toute procédure pénale. Elle représente un levier fondamental de la régulation du système pénal et de la répression des crimes puisqu'elle détermine le nombre et le type d'affaires qui seront renvoyées devant les tribunaux. Les législateurs de 1791 ont pleinement conscience de l'enjeu que constitue le contrôle de la poursuite et se montrent méfiants à l'égard de l'usage que pourrait en faire le roi et de manière plus générale le gouvernement. Ils décident par conséquent de déléguer la poursuite à des officiers de police judiciaire élus, en l'occurrence les juges de paix, les directeurs du jury d'accusation et les accusateurs publics. Dans cette configuration, les pouvoirs des représentants du gouvernement (appelés « commissaires du gouvernement ») se retrouvent limités et consistent principalement à vérifier la légalité des poursuites et à surveiller l'activité judiciairc.

Au début du Consulat, l'organisation de la poursuite établie par les Constituants fait l'objet d'une réforme radicale à travers le vote de la loi du 7 pluviôse an IX. Cette loi confie désormais le pouvoir de poursuite aux commissaires du gouvernement tandis qu'elle limite la compétence des juges de paix et des directeurs du jury à la seule instruction. La fonction d'accusateur public, quant à clle, disparaît et ses pouvoirs sont délégués au représentant du gouvernement (art. 63 titre $\mathrm{V}$ de la Constitution de l'an VIII). Les raisons avancées pour justifier le transfert de la poursuite des mains de juges élus à celles des magistrats nommés par les Consuls consistent à accuser les officiers de police judiciaire élus d'inactivité, de lenteur, d'indulgence ou de collusion avec la population. Cette inertie cumulée à l'influence des passions locales sur l'exercice des poursuites engendrerait dès lors l'impunité du criminel et serait à l'origine de la déstabilisation de l'ordre public.

De telles critiques sont en partie fondées. À travers l'étude de l'activité de poursuite des officiers de police judiciaire, nous avons constaté qu'il existe une collusion avec les populations ou une réticence évidente à poursuivre certaines infractions. Il reste cependant à évaluer l'ampleur de l'inertie ou de l'indulgence des officiers de police judiciaire et à déterminer si ce comportement constituait un danger pour le maintien de l'ordre public.

Pour répondre à cette question, nous avons pris comme indicateur le taux d'abandons des poursuites qui équivaut au taux de classement sans suite actuel ${ }^{22}$. En moyenne, $41 \%$ des affaires sont abandonnés mais

(22) Jusqu'à présent, aucune étude n'a été consacrée a l'exercice de la poursuitc sous la Révolution. L'absence de recherches tient essenticliement à la difficulté de découvrir des sources équivalentes aux registres du parquet du XIX siècle qui permettent de suivre l'activité de poursuite des magistrats. Par chance, ce type de sources a été conservé pour quatre arrondissements judiciaires des départements belges entre l'an IV et l'an VIII : Bruxelles intérieur (Dyle), Bruxelles extérieur (Dyle), Jodoigne (Dyle) et 
ce taux varie fortement en fonction des types d'infractions. Les vols et les homicides qui sont susceptibles de porter directement atteinte à la sûreté des citoyens comptent parmi les infractions dont les taux d'abandons de poursuites sont parmi les plus faibles : $24 \%$ d'abandons pour les vols ${ }^{24}$ et $21 \%$ pour les homicides ${ }^{25}$. En réalité, les taux d'abandons de poursuites les plus ćlevés sont monopolisés par les infractions que l'on qualifie de politiques telles que les infractions à la police des cultes ${ }^{26}$ (59\% d'abandons de poursuites $)^{27}$ ou les crimes contre-révolutionnaires ${ }^{2 \pi}$ (56\% d'abandons des poursuites $)^{29}$. Or ces infractions politiques se singularisent par le fait que la légitimité de leurs poursuites est fortement contestée, comme en témoigne les crimes d'attroupements séditieux.

Larticle 5 de la loi du 27 germinal an IV définit comme attroupement séditieux, tout rassemblement où l'on provoquerait la dissolution du gouvernement républicain et où l'on attenterait à la sûreté publique et individuelle. L'article 6 de la loi prévoit des peines qui varient, suivant le statut des prévenus, de 5 années de fers à la peine de mort. Divisé entre les intérêts d'une population dont il est l'émanation et l'exécution de la loi, le juge de paix ou son supérieur, le directeur du jury, jouent un rôle temporisateur et ne poursuivent que les infractions les plus graves. Cette attitude est nécessitée par le caractère controversé des dénonciations et des arrestations opérées par les différentes autorités. Celles-ci peuvent considérer un simple attroupement comme séditieux et ordonner des arrestations qui s'avèrent infondées. Larbitraire des poursuites prend, dans certains cas, des proportions surréalistes. Laffaire de l'orchestre de Mons (département de Jemappes) constitue, de ce point de vue, un exemple abouti ${ }^{30}$.

Mons (Jemappes). Il s'agit des " registres d'entrée au greffe " du directeur du jury décrivant chaque affaire poursuivie par un officier de police judiciaire et transmisc au greffe du directeur du jury. À partir des registres, nous pouvons identifier l'officier de police judiciaire ayant instruit l'affaire, le prévenu, te type d'infraction et l'issue des poursuites. Ces données quantitatives sont complétées par des informations plus qualitatives contenues dans les dossiers de procédure leur correspondant. La présente étude a été réalisée à partir de ces registres d'entrée au greffe. Les archives de l'arrondissement judiciaire de Bruxelles sont conservées aux Archives de l'État à Anderlecht (Fonds du tribunal de $\left.\right|^{\text {cr }}$ instance de Bruxelles $n^{\circ} 117$ et $\mathrm{n}^{\circ}$ 199), les archives de l'arrondissement judiciaire de Jodoigne sont conservées aux Archives de l'État du Brabant wallon (fonds du tribunal correctionnel de Nivelles, $\left.n^{\circ} 140\right)$ et les arehives de l'arrondissement judiciaire de Mons sont conservées aux Archives de l'État à Mons (fonds du tribunal de correctionnel de Mons, $n^{\circ} 10$ et 11 ).

(23) 1768 affaires sur un total de 4285 .

(24) 249 affaires sur un total de 1032.

(25) 37 affaires sur un total de 175.

(26) Loi du 7 vendémiaire an IV.

(27) 44 affaires sur un total de 74 .

(28) Les crimes contre-révolutionnaires poursuivis sous le Directoire devant les juridictions ordinaires sont principalement composés des propos, écrits et attroupements séditicux (loi du 27 germinal an IV) et des atteintes portées à l'arbre de la liberté (loi du 24 nivôse an VI).

(29) 55 affaires sur un total de 98 . $n^{\circ} 32 / 152$.

(30) Archives de l'État à Mons, fonds du tribunal correctionnel de Mons, dossier de procédure 
Le 4 brumaire an V, le juge de paix de la $1^{\text {ire }}$ section de Mons, Jean-Baptiste Deghilage, reçoit de la part de Senault, commandant temporaire de la place, une dénonciation alarmiste :

«Des personnes dignes de foi m'ayant informé que des rassemblements armés ayant à leur tête une musique militaire, se formaient en cette place, aux bruits des airs guerriers et en parcouraient les rues; que les individus qui les composaient étaient des amis de la maison d'Autriche, qui tenaient des propos insidieux tendant à un soulèvement. Hier soir vers 10 heures ayant été averti de nouveau que cè rassemblement commençait à se former et que le signal était donné, j’envoyai de suite, par mon secrétaire, l'ordre au chef du poste de la grand'garde de l'accompagner avec une partie de sa garde et d'arrêter indistinctement tous rassemblements quelconques ; ce qu'ils firent. Je vous envoie ci-joint le rapport du chef de ce poste [...] ainsi que les individus arrêtés. Veuillez ne rien négliger pour découvrir leurs intentions sinistres. Ils peuvent être les autcurs des cocardes autrichiennes semées, des affiches incendiaires placardées, des bruits alarmants jetés dans le public contre la République française et ses défenseurs, enfin dans les circonstances actuclles, ces individus avaient certainement des vues et des projets dangereux. Je vous prie de faire mention dans votre procès-verbal des instruments dont ces individus sont porteurs, ce sont des preuves de délits, tels que caisse roulante, grosse caisse, tambour de basse etc ».

Les 4 et 5 brumaire, le juge de paix effectue les visites domiciliaires demandées et interroge les vingt citoyens arrêtés. Au fil des perquisitions et des déclarations, le juge de paix doit cependant reconnaître l'absence de fondement de la dénonciation. Les prévenus s'étaient réunis pour jouer de la musique dans l'intention de se divertir en l'honneur de la Saint-Crépin. Accusés d'exécuter « des marches et airs proscrits comme la marche des Brabançons des ci-devant États et de Ô Richard Ô mon Roi " et de tenir «dans les endroits où ils se trouvaient des propos inciviques et tendant à la révolte ", les prévenus et les témoins répondent qu'ils ne jouent que des airs d'opéra. Les doubles aigles, armoiries de la maison d'Autriche, qui décoraient les grosses et petites caisses, de même que « les autres objets relatifs à l'armement et attroupement avec musique guerrière » ne sont pas retrouvés. Un bonnet « aux coulcurs autrichiennes ou des ci-devant États [de Brabant] ", porté par l'un des attroupés, se révèle être un bonnet turc à fond jaune, ligné noir et rose et garni de perles blanches, qui est utilisé lors des farces populaires.

Confronté à des réponses difficilement réfutables, le juge de paix en vient à demander aux prévenus « de nous déclarer sincèrement pourquoi cette musique guerrière s'augmentait chaque jour et pourquoi on s'y exerçait aussi souvent si ce n'était pas pour faire parade à l'époque à laquelle les ennemis de la République auraient quelque succès, j'entends avec 
lesquels tous les chefs et auteurs de cette musique, depuis longtemps connus par leur incivisme, savaient tromper les dupes pour mieux cacher leurs mauvaises intentions ". La réponse est cinglante : les répétitions se font deux fois par semaine et l'orchestre se produit les jours de fête. L'un des prévenus, Claustriau, remet au juge de paix la permission écrite du commandant Senault, leur dénonciateur, en date du 7 frimaire an IV, qui autorise le " citoyen Claustriau de se divertir chez lui et près de chez lui avec de la musique et un tambourinet ".

Face à cette autorisation, le juge de paix perd visiblement le fil des débats et demande « à quoi pouvait dans les circonstances actuelles servir une grosse caisse et tant d'autres instruments formant une musique guerrière quand on n'a que des intentions aussi simples que de s'amuser ". Charles Amand, un autre prévenu, répond tout bonnement "qu'il croyait que c'était à cause que beaucoup parmi eux ignorant la musique et désirant s'amuser avaient préféré de battre unc caisse ou autres instruments futiles". Doutant de la pertinence des poursuites, le juge de paix demande au commandant de la place Senault de lui livrer les noms des dénonciateurs mais celui-ci refuse catégoriquement, mettant en doute la puissance cœrcitive de la justice.

Le refus du commandant n'empêche cependant pas le juge de paix de continuer l'instruction. Il obtient notamment de la part de Senault des explications sur les origines des événements. D'après le commandant, plusieurs militaires de service lui ont déclaré que vers 22 heures, le 3 brumaire an $V$, " l'on tirait du canon aux environs de la place, que sur ce [le commandant Senault] s'était porté sur le rempart près de la tour de Saint-Nicolas, pour s'assurer de la vérité, qu'en effet il entendit des bruits sourds, qui lui parurent l'explosion du canon, que de suite il donna l'ordre à la garnison de prendre les armes et rester dans leurs casernes respectives jusqu'à nouvel ordre, mais il ordonna pour empêcher qu'on ne trouble le repos public à plusieurs patrouilles de parcourir les rues dans le plus grand silence et d'arrêter tout rassemblement quelconque ».

Si l'on en croit le commandant, toute l'affaire trouverait son origine dans la confusion des bruits de grosse caisse avec des bruits de canon. S'agit-il d'un subterfuge destiné à faire cesser une fête populaire trop bruyante et à couvrir des arrestations qui s'avèrent désormais arbitraires? Les moyens utilisés semblent dans tous les cas disproportionnés, d'autant que Senault avait autorisé l'orchestre. À la suite de la déclaration du commandant, le juge de paix ne semble d'ailleurs plus donner de crédit aux allégations d'" attroupements nocturnes munis d'instruments formant une musique guerrière, $[\ldots]$ prévenus des intentions contre-révolutionnaires tendant à troubler la République et la tranquillité publique ». Laffaire est renvoyée au directeur du jury de Mons qui met l'ensemble des prévenus en liberté. 
De telles affaires prêtent aujourd'hui à sourire mais il est certain qu'à l'époque ces procédures n'amusent pas grand monde au sein de la population. Pour avoir jouć des airs d'orchestre un jour de fête, vingt citoyens sont arrêtés et poursuivis pour attroupement séditieux, un crime passible de la peine de mort. L'exemple de l'orchestre de Mons n'est pas isolé, on compte des dizaines d'affaires semblables dans lesquelles des individus ayant crié dans un cabaret "Vive l'empereur François II " ou «Merde à la République» sont susceptibles d'être guillotinés. En réalité, dans ce genre de dossier, l'inertie ou l'indulgence des officiers de police judiciaire ne mettent pas en danger la sûreté de la société mais tendent au contraire à protéger les libertés individuelles face à des dénonciations abusives et une peine disproportionnée par rapport aux faits.

\section{La mise en accusation aux mains des jurys populaires}

Le jury d'assises actuel constitue le dernier témoignage de la participation directe des citoyens à l'exercice de la justice. S'il réussit à survivre aux différents changements de régime, on oublie généralement que les Constituants avaient décrété, sur le modèle du Grand Jury anglais, l'établissement d'un premier jury au stade de la mise en accusation. Ce dernier est composé de citoyens qui décident s'il existe des présomptions suffisantes pour renvoyer le prévenu devant le jury de jugement du tribunal criminel du département. Les deux jurys sont alors conçus comme indissociables.

Linstitution du jury en matière criminelle est adoptée le 30 avril 1790. Son introduction constitue la pierre angulaire de la nouvelle organisation judiciaire. La protection des libertés individuelles exige en effet que les citoyens accusés de crimes soient jugés par leurs pairs. Dans son discours sur l'ordre judiciaire en date du 29 mars 1790, Adrien Duport justifie cette exigence. Le député y rappelle que le principe suivant lequel " tous les pouvoirs sont établis par le peuple et pour le peuple $»^{31}$ implique que le peuple ne délègue pas les pouvoirs qu'il peut assumer lui-même. Or, à travers le jury, les citoyens ont la possibilité d'exercer directement leur souveraineté et de fonder la base du pouvoir judiciaire. Ils ne craindront dès lors plus ni le despotisme du gouvernement ni celui des tribunaux. Le jugement par jurés sera regardé comme « le boulevard de la liberté individuelle $n^{32}$.

$\mathrm{Si}$, tout au long de Révolution et du Consulat, le jury est qualifié de « palladium des libertés », des critiques s'élèvent à l'encontre de son indulgence et des acquittements dits scandaleux. Les attaques sont similaires à

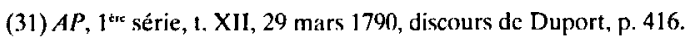

(32) Idem. 
celles émises envers les juges de paix. Les jurés seraient incompétents ou corrompus et leur indulgence, en engendrant l'impunité du crime, déstabiliserait l'ordre social. Cette défiance aboutira à la suppression du jury d'accusation le 6 février 1808 et à son remplacement par une chambre des mises en accusation dépendant de la Cour d'appel. Toutefois, la longueur des discussions, la diversité des opinions et la contradiction des discours qui précédèrent la suppression du jury d'accusation indiquent qu'elle n'allait pas de soi. Malgré les critiques formulées à l'égard des « acquittements scandaleux ", l'idée et la fonction du jury d'accusation semblent avoir été mieux acceptées que ses détracteurs n'aient voulu l'admettre. Aussi, avonsnous cherché à examiner le fondement des critiques émises à l'encontre du jury d'accusation à travers l'analyse de son activité sous le Directoire.

À l'image de ce que nous avons constaté pour les officiers de police judiciaire, les attaques sont partiellement fondées mais l'indulgence des jurés varie suivant les types d'infraction ${ }^{33}$. Le taux moyen d'acquittements prononcés par les jurés d'accusation est de $33 \%$ mais il n'est que de $26 \%$ pour les homicides ${ }^{35}$ et de $23 \%$ pour les vols qualifiés ${ }^{36}$. En réalité, les " acquittements scandaleux " touchent principalement les crimes politiques dont $54 \%$ des dossiers aboutissent à la mise en liberté des prévenus ${ }^{37}$. On constate par conséquent que les jurés d'accusation prolongent l'action des officiers de police judiciaire et que les affaires politiques soumises au jury posent à nouveau la question de la légitimité des poursuites. Le cas des affaires de complicité d'évasion constitue à cet égard un bel exemple.

Pendant tout le Directoire, le gouvernement est confronté à un nombre extrêmement élevé d'évasions. Celles-ci s'expliquent par la vétusté des prisons, le manque de personnel et l'inertie du gouvernement et des administrations départementales à y remédier. Cette conjoncture est directement liée au bouleversement introduit par l'invention de la prison pénale sous la Constituante. Dans une volonté généreuse d'humaniser les peines, les législateurs ont substitué à « l'arbitraire » et à la « barbarie » des châtiments corporels de l'Ancien Régime, un nouveau système pénal établi sur l'emprisonnement. Mais rapidement, les prisons d'Ancien Régime se trouvent submergées et totalement inadaptées à leurs nouvelles fonctions. Les conditions matérielles de l'application des principes pénaux révolutionnaires s'avèrent impraticables compte tenu de l'absence de lieu spécifique

(33) Leétude a été réalisée à partir des documents utilisés dans le cadre de la poursuite : les registres d'entrée au greffe du directeur Ju jury daccusation des arrondissements de Bruxelles (Dyle), de Jodoigne (Dyle) et de Mons (Jemappes) entre l'an IV et l'an VIII. Nous avons ajouté les données des registres des jurys d'accusation de l'arrondissement de Turnhout (Deux-Nèthes) conservés aux Archives de l'État à Beveren (Fonds du tribunal de $l^{\text {err }}$ instance de Turnhout $n^{\circ} 65$ ).

(34) 284 affaires sur un total de 864 .

(35) 37 affaires sur un total de 140.

(36) 98 affaires sur un total de 419.

(37) 101 affaires sur un total de 188. 
pour enfermer les détenus et du défaut de moyens financiers pour y suppléer ${ }^{38}$.

Les autorités ont pleinement conscience de la situation ${ }^{34}$. Plusieurs messages du Directoire et les rapports de commissions nommées par le Conseil des Cinq-Cents soulignent l'état catastrophique des prisons dans la plupart des départements. Mais la crise que traverse la France de l'an III à l'an $\mathrm{V}$ empêche toute amélioration. Les fonds sont prioritairement absorbés par la solde et la subsistance des armées. Viennent ensuite les rentes, les pensions et le traitement des fonctionnaires. Il ne reste dès lors pratiquement plus rien pour éponger les dépenses non prioritaires telles que ceiles des prisons. Sans argent, les réparations les plus urgentes sont reportées sine die, les geôliers ne sont plus payés, les fournisscurs se retirent, des famines se déclarent dans les prisons. À partir de l'an VI, une légère embellie budgétaire apparaît du fait des victoires remportées par les armées républicaines, de l'amélioration des finances de l'État et de l'arrivée d'un ministre de l'Intérieur compétent, François de Neufchâteau. Mais l'éclaircie financière est principalement affectée au règlement des arriérés et n'améliore guère l'ordinaire des détenus ni l'état des prisons.

À défaut de pouvoir libérer les moyens financiers nécessaires à l'entretien des prisons, le Directoire décide de renforcer le contrôle du personnel chargé de la garde des détenus par le développement d'une politique répressive à leur encontre. À cet effet, le gouvernement obtient, le 4 vendémiaire an VI, le vote d'une loi prévoyant une peine maximale de 12 années de fer à l'égard des individus dont la négligence a permis l'évasion des détenus. Ladoption de la loi du 4 vendémiaire an VI ne produit cependant pas les résultats espérés. Non seulement le flux d'évasions ne semble pas s'infléchir mais surtout les prévenus ne sont que rarement jugés par les tribunaux criminels. La raison tient au rôle du jury d'accusation qui acquitte massivement les gardiens de prison et les gendarmes.

De l'an IV à l'an VIII, la complicité d'évasion est l'un des crimes dont les auteurs sont les plus fréquemment acquittés $(77 \%)^{40}$. Si ce taux incroyablement élevé tend au premier abord à conforter les accusations d'impunité proférées par le Directoire à l'encontre des jurés populaires, il relève en réalité de facteurs objectifs déjà évoqués, qui mettent le gouvernement face à ses responsabilités et questionnent sa logique répressive.

Limportance prise par le manque de personnel et le délabrement des prisons comme facteurs explicatifs de la fuite des détenus indique en effet que la négligence et la connivence des gardiens sont loin d'être

(38) Marie-Sylvie Dupont-Bouchat, a Prisons et prisonniers à Namur sous le Régime français », Annales de la société archéologique de Namur, 1998, t. 72, p. 343-388.

(39) Jacques-Guy PETIT, Ces peines obscures. La prison pénale en France (1789-1875), Paris, Fayard, 1990, p. 93-108.

(40) 27 affaires sur un total de 35 . 
établies. Il paraît difficile pour les jurés d'accusation de rendre ces derniers responsables des évasions alors qu'elles sont principalement provoquées par la lenteur de l'administration et par la crise financière. Le malaise à l'égard de l'accusation des gardiens est d'autant plus fort que ceux-ci essaient, dans la mesure du possible, de rattraper les fugitifs. Le concierge de la maison de police de l'Amigo, Van Asse, entame le 6 pluviôse an VI des poursuites dans les rues de Bruxelles accompagné de deux chasseurs ${ }^{41}$; le gardien en chef de la maison centrale de détention de Vilvorde, Van Buggenhout, réussit, lors de l'évasion du 27 nivôse an VII, à rattraper quatre des neuf fugitives ${ }^{42}$; le 15 fructidor an VIII, le nouveau gardien de Vilvorde, De Witte, et plusieurs vétérans mènent sans succès des recherches aux alentours de la maison de détention ${ }^{43}$, etc.

La bonne volonté dont font preuve les gardiens dans la recherche des évadés ne doit cependant pas occulter certains comportements douteux, susceptibles d'être assimilés à la négligence ou à la collusion dénoncées par le Directoire. Ces accusations sont alimentées par la proximité des gardiens et des détenus et par le caractère bon enfant de leurs relations qui rappellent les habitudes de l'Ancien Régime. Le cas le plus extrême concerne Alexis De Witte, alors qu'il occupait les fonctions de geôlier à la maison de détention de la porte de Hal (département de la Dyle) ${ }^{4}$. Le 12 thermidor an IV, vers trois heures du matin, De Witte quitte Bruxelles en voiture accompagné de l'ancien mayeur de Longueville, Dezonne, condamné à quatre années de détention. Les deux protagonistes se rendent dans la cense du détenu située au-dessus de Wavre. En acceptant le voyage, De Witte répond aux sollicitations continues de Dezonne qui « désirait ardemment voir sa mère qui serait fortement malade ». Ils n'y restent que le temps de dîner et reviennent à Bruxelles à 4 heures et quart. Entre-temps, l'escapade est connue et produit le plus mauvais effet.

Lalerte est donnée par le portier de la porte de Hal qui refusa l'escalin que De Witte lui proposait pour le laisser passer. La voiture réussit finalement à sortir par la porte de Louvain. À 3 heures, le commissaire près la municipalité, Rouppe, reçoit de la part du commandant de la garde de la municipalité un rapport alarmiste suivant lequel « le geôlier de la porte de Hal et deux autres personnes s'étaient échappés par une des portes de cette ville, après avoir tenté de le faire par celle de Hal, et qu'on avait vu un attroupement et entendu des coups de feu hors de la porte ; et vu entrer plusieurs personnes dans la prison la nuit ». Rouppe se rend immédiatement à la maison de détention et interroge la femme du geôlier sur la

(41) Archives de l'État à Anderlecht, fonds du tribunal correctionnel de Bruxelles, dossier de procédure ${ }^{\circ} 25 / 758$.

(42) $\mathrm{lbid}$, dossier de procédure $n^{\circ} 45 / 723$.

(43) libid., dossier de procédure $n^{\circ} 39 / 1246$.

(44) Ibid., dossier de procédure $n^{\circ} 7 / 315$ 
raison de son silence. Celle-ci lui répond " qu'elle croyait que c'était pour le bien qu'il le faisait et qu'elle n'irait pas faire des rapports contre son mari ». À la demande de l'accusateur public, le juge de paix de la $1^{\text {ere }}$ section de Bruxelles procède à la visite de l'ensemble des cachots afin de prendre les mesures conservatoires et nomme provisoirement l'adjoint Altenhove concicrge de la porte de Hal. Toute cette effervescence ne prend fin qu'au retour du cabriolet. Interrogé le lendemain par le juge de paix quant aux raisons de sa fuite, De Witte explique qu'il n'a accepté le voyage que "par humanité ". Il n'a d'ailleurs rien reçu en échange si ce n'est une douzaine de gaufres pour ses enfants. Quant au danger que son départ est susceptible de causer pour la sûreté de la prison, il assure " qu'il a laissé sa femme avec les domestiques pour surveiller les prisonniers et que par conséquent il n'y avait point de danger, qu'il n'a fait en cela que ce qu'il faisait journellement quand il allait en commission ».

Le retour du détenu quelques heures après son " évasion » n'empêche pas De Witte d'être suspendu de ses fonctions et d'être prévenu d'avoir favorisé la fuite de l'ancien mayeur. Indépendamment des motifs humanitaires et de l'absence de danger pour la sécurité de la maison de détention, la connivence de De Witte est clairement établie. Une telle évidence est exceptionnelle et est difficilement décelable dans les autres dossiers. Seules les affaires impliquant des gendarmes donnent le spectacle d'une négligence aussi manifeste. Lévasion se produit généralement parce que l'individu arrêté est mal ou pas du tout attaché. Les gendarmes ne nient pas le fait mais tentent de se justifier. Le gendarme Jean-Baptiste Garnotel avance que lorsque le juge de paix lui demanda de conduire François Verdoot à la prison de l'Amigo, il ne crut pas nécessaire de le garrotter dans la mesure où aucun mandat d'arrêt n'avait été dressé. Le juge de paix ne lui a en outre pas déclaré la nature du délit (il s'agit d'un vol aggravé) et il n'a pas pu le comprendre au cours de l'interrogatoire vu qu'il se déroulait en flamand. À l'approche de l'Amigo, François Verdoot prend la fuite vers la Grand-Place et le gendarme, à cheval, ne parvient pas à le rattraper étant donné l'encombrement provoqué par les boutiques de la foire. Garnotel tente ensuite de le retrouver et interroge les passants mais ceux-ci lui répondent en flamand «ik kan niet verstaen " (« je ne peux pas comprendre »)

La déclaration de Garnotel insinue que la foule s'est montrée complice de l'évasion. Il s'agit du principal argument avancé par les gendarmes afin de fuir leur responsabilité. Il est notamment invoqué par deux gendarmes et six fusiliers jugés pour avoir facilité l'évasion d'AlbertPhilippe Gilbert. Le 14 thermidor an VI, le tribunal correctionnel de Bruxelles prononce neuf condamnations de deux années d'emprisonnement et de dix francs d'amende à l'encontre d'une bande d'escrocs. À la 
sortie de l'audience, les gendarmes constatent qu'ils n'ont pas suffisamment de corde pour lier tout le monde mais décident néanmoins de procéder au transfert des condamnés. Alors que ces derniers sont conduits dans les voitures placées devant la maison du peuple, l'un d'entre eux, AlbertPhilippe Gilbert, réussit, libre de ses mouvements, à sauter par dessus l'escalier et à se fondre dans la foule. Face au directeur du jury, les gendarmes et les militaires refusent d'endosser une quelconque responsabilité. Si l'évasion a pu se produire, c'est avant tout à cause de la « très grande affluence de monde; que ceux qui composaient cette réunion étaient presque tous des personnes de la troupe des condamnés, qui firent des mouvements pour causer du désordre à l'effet de favoriser l'évasion desdits condamnés; que pendant que les gendarmes se mettaient en garde contre ces tentatives criminelles, un des condamnés, Albert-Philippe Gilbert $[\ldots]$ se mêla à la foule et au moyen de la protection qu'il y trouva, parvint à s'évader $n^{40}$.

La négligence des gendarmes, pas plus que la connivence du geôlier De Witte, ne provoquent de mise en accusation. Les jurés ne leur tiennent visiblement pas rigueur de leurs fautes. Ils se montrent aussi indulgents que lorsque les évasions sont provoquées par le manque de personnel ou par la vétusté des prisons. La raison de l'indulgence est évidente. Larrestation d'un geôlier rend la prison difficilement gérable en son absence et risque de provoquer de nouvelles évasions. À l'égard des gendarmes, il est probable que les jurés tiennent un raisonnement similaire : qui escortera les condamnés et assurera la sécurité de tous si l'on emprisonne les gendarmes ? Cette prise de conscience de la difficulté de l'administration carcérale et de la faiblesse de ses moyens explique la raison pour laquelle les jurés d'accusation n'hésitent pas à acquitter à plusieurs reprises le même gardien de prison. C'est le cas à Turnhout où le geôlier de la maison d'arrêt de l'arrondissement, Anselme Caeluwaerts, est acquitté les 30 thermidor an VI, 10 vendémiaire an VII, 30 thermidor an VII et 28 thermidor an VIII ${ }^{4}$. Dans l'arrondissement de Jodoigne, le gardien de la maison d'arrêt est acquitté les 16 frimaire an V, 22 germinal an VII et 25 frimaire an VIII ${ }^{48}$. La plupart des gardiens réoccupent ensuite leur fonction. Ces réintégrations indiquent que les autorités administratives, pas plus que les jurés d'accusation, ne tiennent à accentuer les difficultés de gestion des prisons.

Si dans les affaires de complicité d'évasion, la collusion des jurés avec le prévenu est établie, elle ne vise pas à déstabiliser l'ordre public mais à adapter des politiques et des lois pénales contestées aux réalités du terrain. Lexercice de la justice par le peuple souverain constitue par consé-

(46) lbid., dossier de procédure $n^{\circ} 45 / 900$.

(47) Archives de l'État à Beveren, fonds du tribunal de $1^{\text {th }}$ instance de Turnhout, $n^{\circ} 65$.

(48) Archives de l'État du Brabant wallon, fonds du tribunal correctionnel de Nivelles, $n^{\circ} 140$. 
quent une sorte de garde-fou à l'exécution immodérée et inutile de lois répressives. Ce comportement sans doute perçu comme un abus de pouvoir, peut également apparaître comme légitime aux contemporains. Lors de la discussion de la loi du 4 vendémiaire an VI relative à la complicité d'évasion, le Corps législatif avait conditionné l'adoption de la résolution à la réfection et au refinancement préalable des prisons. Un tel manquement donnerait, aux yeux du rapporteur Siméon, " un prétexte si plausible à [l']évasion [des détenus], ou rendrait cruelle et presque criminelle la surveillance de leur gardien ${ }^{49}$.

Cette réalité n'empêche cependant pas l'accentuation des critiques à l'encontre du jury populaire. Le jury d'accusation est finalement supprimé après beaucoup d'hésitations le 6 février 1808 mais il ne s'agit là que de la date de sa mort clinique. Le jury a en effet été vidé de son sens, huit ans plus tôt, sous le Consulat. Le 4 pluviôse an IX, le Corps législatif décide que les jurés d'accusation ne se prononceront plus d'après les déclarations orales des témoins mais uniquement d'après leur déclaration écrite. Lintroduction de la preuve écrite est perçue par certains législateurs comme une "atteinte mortelle " s" au jury d'accusation et un retour aux preuves légales de l'Ancien Régime. Selon le tribun Bouteville, le juré d'accusation sera relégué à un rôle de mannequin ridicule qui ne se prononcera plus d'après sa conscience mais d'après ce que l'officier de police judiciaire aura bien voulu lui exposer. Un autre orateur, Ganilh, soutient que la nouvelle loi est un «mélange impur : il ne peut y avoir d'alliance entre les formes oppressives de la monarchie et les formes protectrices de la République. Ces formes se repoussent mutuellement, et ne peuvent pas concourir au même but $\aleph^{5 /}$.

Malgré l'adoption de la réforme du jury d'accusation, plusicurs législateurs se montrent par conséquent lucides par rapport aux implications de la nouvelle loi. Ils refusent de brader l'organisation judiciaire de 1791 et de mettre en danger les libertés individuclles au nom de la restauration de l'ordre public. A posteriori, ces mises en garde s'avèrent justifiées au regard non seulement de l'évolution autoritaire du régime napoléonien mais surtout de l'activité des organes judiciaires populaires.

On constate tout d'abord que les vols et les homicides qui portent directement atteinte aux droits naturels des citoyens comptent parmi les infractions dont les taux d'abandons de poursuites et d'acquittements sont parmi les plus faibles. En réalité, l'indulgence et la collusion des juges et des jurés d'accusation concernent principalement les infractions politiques

(49) AN. AD XVIIIc 396, rappont fait par Siméon sur deux messages du Directsire exécuif, relatifs à l'évasion des détenus, en date du 8 ventôse an $\mathrm{V}$.

(50) $A P, 2$ série, t. II, 3 pluviôse an IX, discours de Chabot (de l'Allier), p. 154.

(51) Ibid., t. II, 2 pluviôse an IX, discours de Ganilh, p. 137. 
dont la légitimité des poursuites est la plus contestée. D'autre part, les crimes les plus graves tels que le brigandage, les assassinats sur les grands chemins ou les révoltes armées ne sont généralement pas soumis à la justice ordinaire mais sont jugés par des juridictions d'exception telles que les Conseils de Guerre et les Commissions militaires.

Ces éléments laissent penser que les réformes judiciaires du Consulat et de l'Empire n'ont pas pour unique objectif de rétablir l'ordre public. Elles visent également à soumettre l'indépendance de la justice et les principes libéraux du modèle judiciaire révolutionnaire aux législations et aux politiques pénales du gouvernement. La soumission du pouvoir judiciaire n'est dès lors plus seulement conçue pour assurer le maintien de l'ordre public mais également pour servir un nouvel ordre politique fondé sur la domination du gouvernement

Si la réussite ou l'échec du modèle judiciaire libéral en matière de maintien de l'ordre public sont sujets à débats, il n'en est pas de même pour la question de la protection des libertés individuelles. La disproportion des peines, les dénonciations arbitraires et la mise en ceuvre de politiques répressives illégitimes constituent des atteintes que les Constituants cherchaient à prévenir. Face à de tels abus, les juges et les jurés prononcent des mises en liberté afin de préserver les intérêts et les droits naturels des citoyens. De ce point de vue, le modèle judiciaire de la Révolution constitue une réussite.

Emmanuel BERGER

FNRS / UCL Louvain-la-Neuve

Centre d'histoire du droit et de la justice rue du Poirier, 10

B-1348 Louvain-la-Neuve

Belgique

emmanuelberger@hotmail.com 